Figure 1 a)

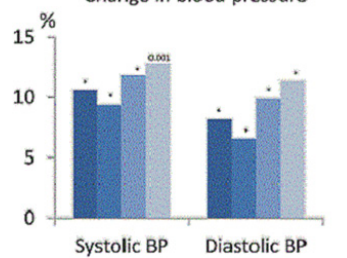

valoe $\infty 00,0001$ Change in blood pressure

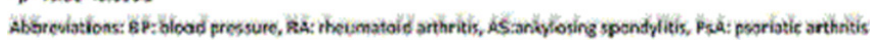

a-HTT was changed. Systolic BP $(p<0.0001)$ was significantly associated with BP goal attainment in age- and sex adjusted logistic regression analyses, while the use of anti-rheumatic medication or inflammatory biomarkers at baseline was not. Patients with the lowest systolic BP were more likely to achieve BP goals. For patients already on a-HTT $(n=224)$, only $52.7 \%$ had a BP $<140 / 90 \mathrm{mmHg}$ at baseline. After up titration or change of a-HTT, the percentage of patients achieving BP goal in this group increased to $82.6 \%$.

Conclusions: This is to our knowledge the first prospective report on success rate of $\mathrm{BP}$ goal achievement in patients with IJD. Approximately $80 \%$ reached $\mathrm{BP}$ target, which is even a higher proportion than what is shown in the general population. Treatment to BP goal is feasible in patients with IJD, and is not complicated by inflammation or use of anti-rheumatic medication.

Disclosure of Interest: None declared

DOI: 10.1136/annrheumdis-2017-eular.6937

\section{SAT0091 IS CLINICAL ARTHRITIS ALWAYS PRECEDED BY SUBCLINICAL INFLAMMATION? A LONGITUDINAL STUDY AT JOINT LEVEL IN PATIENTS WITH ARTHRALGIA THAT DEVELOPED ARTHRITIS}

R.M. Ten Brinck, H.W. van Steenbergen, A.H. van der Helm-van Mil. Rheumatology, Leiden University Medical Centre, Leiden, Netherlands

Background: The clinical phase of Rheumatoid arthritis (RA) is preceded by a phase with subclinical inflammation. MRI can detect subclinical inflammation and, at patient level, this is predictive for the development of clinical arthritis. However, at joint level it is unknown how arthritis develops. It is unknown how frequently joints with subclinical inflammation progress to clinical arthritis, and vice versa, how often joints that developed clinical arthritis had local subclinical inflammation during the preceding phase of arthralgia. A longitudinal MRI study in patients that developed arthritis can unravel if arthritis development is restricted to some locations in which the severity of inflammation increases over time or, alternatively, if the process is more generalized with a weak association between the locations of subclinical inflammation and subsequent clinical arthritis.

Objectives: This longitudinal study at joint level during progression from pre-RA to RA determined the relation between the location of subclinical inflammation and clinical arthritis over time.

Methods: 290 small joints (4 MCPs, 1 wrist, 5 MTPs per person) of 29 patients that presented with arthralgia and developed clinical arthritis were studied with 1.5T MRI at both time-points. MRIs were evaluated for BME, synovitis and tenosynovitis by three readers (ICCs $0.98,0.96$ and 0.97 ) that were blind to clinical data and the order in time. Subclinical inflammations was defined as presence of BME, synovitis and/or tenosynovitis.

Results: The median time between presentation with arthralgia and clinical arthritis development was 17 weeks. At presentation with arthralgia 68 joints had subclinical inflammation and no significant association was found between joint tenderness and the presence of local MRI-detected subclinical inflammation (OR $0.98 ; 95 \% \mathrm{Cl} 0.48-1.9)$. Over time, $21 \%$ of 68 joints had resolution of subclinical inflammation, $60 \%$ had persistent subclinical inflammation and $19 \%$ developed

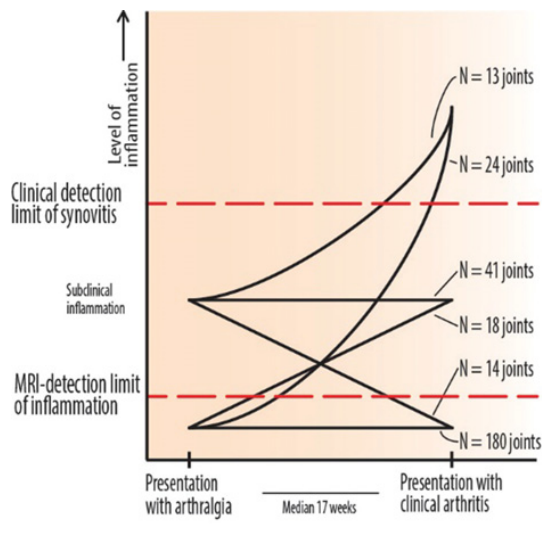

clinical arthritis. At arthritis development 37 joints were swollen. Of these, 24 $(65 \%)$ had no prior subclinical inflammation at the time of presentation with arthralgia (Figure).

Conclusions: This first longitudinal MRI-study on joint level in pre-RA suggested that the majority of joints that developed clinical arthritis had no (long-lasting) preceding phase with subclinical inflammation.

Disclosure of Interest: None declared

DOI: 10.1136/annrheumdis-2017-eular.4693

\section{SAT0092 PROGRESSION OF ATHEROSCLEROSIS OVER 5 YEARS IN A COHORT OF EARLY INFLAMMATORY ARTHRITIS PATIENTS: RESULTS FROM THE NORFOLK ARTHRITIS REGISTER}

S. Skeoch ${ }^{1}$, M. O'Sullivan ${ }^{1}$, J. Gwinnutt ${ }^{1}$, H. Mirjafari ${ }^{1}$, J. Chipping ${ }^{2}$, H. Edlin ${ }^{3}$ A. MacGregor ${ }^{2}$, S. Verstappen ${ }^{1}$, D. Symmons ${ }^{1}$, I. Bruce ${ }^{1,4} .{ }^{1}$ Centre for Epidemiology, University of Manchester, Manchester; ${ }^{2}$ Norwich Medical School, University of East Anglia, Norwich; ${ }^{3}$ The Department of Radiology; ${ }^{4}$ Manchester NIHR Musculoskeletal Biomedical Research Unit, Central Manchester University Hospitals NHS Foundation Trust, Manchester, United Kingdom

Background: Carotid artery intimal medial thickness (IMT) on ultrasound (US) is a validated measure of subclinical cardiovascular (CV) disease. Rates of IMT progression of $0.001 \mathrm{~cm} /$ year $^{1}$ have been observed in the general population and faster progression is associated with increased $\mathrm{CV}$ risk.

Objectives: To describe change in IMT over 5 years in a cohort of early inflammatory arthritis (IA) patients and the association of IMT with estimated CV risk and IA related factors.

Methods: Between 2004 and 2010, patients enrolling in the Norfolk Arthritis Register (a UK inception cohort of IA patients) aged from 16 to 65 years with symptom duration less than 2 years, were invited to take part in the NOAR CVD sub-study. In addition to standard IA disease assessments at 0,2 and 5 years, traditional CV risk factors were measured at baseline and Framingham based 10-year CV risk score was calculated. IMT was measured in the common carotid arteries at baseline and year 5 using a previously validated US protocol. Patient reported clinical CV events at follow up visits which were then verified by a physician. The associations of baseline CV risk and IA characteristics (tender and swollen joint count, rheumatoid factor, CRP, health assessment questionnaire (HAQ), baseline disease modifying anti-rheumatic therapy use) with baseline IMT $\left(\mathrm{IMT}_{0}\right)$ and change in IMT at year $5\left(\mathrm{IMT}_{\Delta}\right)$ were tested using non-parametric statistics. The association of $\mathrm{IMT}_{\Delta}$ with IA characteristics over time (cumulative joint counts, change in $\mathrm{HAQ}$ ) and medication use during follow up (statins, biologic therapy) with $\mathrm{IMT}_{\Delta}$ were explored using non-parametric statistics.

Results: 201 patients with a median (IQR) age and symptom duration of 51 $(42,58)$ years and $10.4(7.7,14.4)$ months respectively were studied. $143(71 \%)$ subjects were female. Median $\mathrm{IMT}_{0}$ was $0.06(0.05,0.07) \mathrm{cm}$ and median $\mathrm{IMT}_{\Delta}$ at 5 -year follow up period was $0.01(0,0.01) \mathrm{cm}$ with an estimated median annual $\mathrm{IMT}_{\Delta}$ of $0.002 \mathrm{~cm}$. IMT had progressed in $104(51.5 \%)$ patients and regressed in $23(11.4 \%)$ patients by year 5 . The median CV risk score at baseline was $5.6(2.6,10.7) \%$ and was associated with $\mathrm{IMT}_{0}$ but not $\mathrm{IMT}_{\Delta}(r=0.46, p<0.01$; $r=0.03, p=0.73$ respectively). There was no association between baseline IA characteristics and $\mathrm{IMT}_{0}$ or $\mathrm{IMT}_{\Delta}$. There was a trend towards an association between cumulative swollen joint count and $\operatorname{IMT}_{\Delta}(r=0.14, p=0.057)$ but no association with cumulative tender joint count, change in $\mathrm{HAQ}$, statin or biologic use (all $p>0.05$ ). CV events occurred in 5 patients during follow up. Of these patients, 3 had IMT progression, 1 had no change and 1 had IMT regression.

Conclusions: Overall, patients with early IA had a higher than expected rate of IMT progression; a significant proportion however had regression in IMT over time. Trajectories of CV risk may vary within the IA population and identifying protective factors will help to better target strategies for CVD prevention in IA.

\section{References:}

[1] G Howard et al. Carotid artery intimal-medial thickness distribution in general populations as evaluated by B-mode ultrasound. ARIC Investigators. Stroke. 1993;24:1297-1304.

Disclosure of Interest: None declared

DOI: 10.1136/annrheumdis-2017-eular.6583

\section{SAT0093 HIGH TRIGLYCERIDES AND LOW HDL LIPID PROFILE AS A SURROGATE MARKER OF HDL DYSFUNCTION IN RA: POTENTIAL LINKS WITH INFLAMMATION AND OXIDATIVE STATUS}

J. Rodríguez-Carrio $^{1}$, M. Alperi-López ${ }^{2}$, P. López ${ }^{1}$, R. López-Mejías ${ }^{3}$, S. Alonso-Castro ${ }^{4}$, F. Abal ${ }^{5}$, F.J. Ballina-García ${ }^{2}$, M.Á. González-Gay ${ }^{3,6,7}$ A. Suárez ${ }^{1}$. ${ }^{1}$ Area of Immunology, University of Oviedo; ${ }^{2}$ Department of Rheumatology, Hospital Universitario Central de Asturias, Oviedo; ${ }^{3}$ Epidemiology, Genetics and Atherosclerosis Research Group on Systemic Inflammatory Diseases, Hospital Universitario Marqués de Valdecilla, IDIVAL, Santander; ${ }^{4}$ Department of Rheumatology, Hospital de Cabueñes, Gijón; ${ }^{5}$ Centro de Salud Sariego, SESPA, Pola de Siero; ${ }^{6}$ Department of Medicine, University of Cantabria, Santander, Spain; ${ }^{7}$ Cardiovascular Pathophysiology and Genomics Research Unit, University of the Witwatersrand, Witwatersrand, South Africa

Background: the interactions between inflammation and lipid profile in RA are 